\title{
Indirect searches for sterile neutrinos at a high-Iuminosity Z-factory
}

\author{
V. DE ROMERI ${ }^{1 *}$, A. ABADA ${ }^{2}$, S. MONTEIL ${ }^{1}$, J. ORLOFF $^{1}$ and A. M. TEIXEIRA ${ }^{1 \dagger}$ \\ ${ }^{1}$ Laboratoire de Physique Corpusculaire, CNRS/IN2P3 - UMR 6533, \\ Campus des Cézeaux, 24 Av. des Landais, F-63177 Aubière Cedex, France \\ ${ }^{2}$ Laboratoire de Physique Théorique, CNRS - UMR 8627, \\ Université de Paris-Sud, F-91405 Orsay Cedex, France \\ E-mail: deromeri@clermont.in2p3.fr
}

\begin{abstract}
A future high-luminosity $Z$-factory has the potential to investigate lepton flavour violation. Rare decays such as $Z \rightarrow \ell_{1}^{\mp} \ell_{2}^{ \pm}$can be complementary to low-energy (high-intensity) observables of lepton flavour violation. Here we consider two extensions of the Standard Model which add to its particle content one or more sterile neutrinos. We address the impact of the sterile fermions on lepton flavour violating $Z$ decays, focusing on potential searches at FCC-ee (TLEP), and taking into account experimental and observational constraints. We show that sterile neutrinos can give rise to contributions to $\mathrm{BR}\left(Z \rightarrow \ell_{1}^{\mp} \ell_{2}^{ \pm}\right)$within reach of the FCC-ee. We discuss the complementarity between a high-luminosity $Z$-factory and low-energy charged lepton flavour violation facilities.
\end{abstract}

The European Physical Society Conference on High Energy Physics 22-29 July 2015

Vienna, Austria

${ }^{*}$ Speaker.

${ }^{\dagger}$ PCCF RI 15-02, LPT Orsay 15-70 


\section{Introduction}

Several extensions of the Standard Model (SM) add sterile neutrinos to the particle content in order to account for neutrino masses and mixings. These models are further motivated by anomalous (oscillation) experimental results, as well as by certain indications from cosmology (see [1,2] and references therein). The existence of these sterile states may be investigated at colliders: for instance, the case for a high luminosity circular $e^{+} e^{-}$collider (called FCC-ee), operating at centre-of-mass energies ranging from the $Z$ pole up to the top quark pair threshold is being actively studied [3]. Its characteristics should allow to obtain a typical peak luminosity at the $Z$ pole of $\sim 10^{36} \mathrm{~cm}^{-2} \mathrm{~s}^{-1}$. A year of operation at the $Z$ pole centre-of-mass energy would yield $\sim 10^{12} Z$ boson decays to be recorded. Motivated by the design study for such a powerful machine, we investigate the prospects for searches for sterile neutrinos by means of rare charged lepton flavour violating (cLFV) $Z$ decays [4].

\section{Leptonic $Z$ decays in the presence of sterile neutrinos}

Lepton-flavour changing $Z$ decays are forbidden in the SM due to the GIM mechanism [5], and their rates remain extremely small $\left(\sim 10^{-54}-10^{-60}\right)$ even when lepton mixing is introduced. The observation of such a rare decay would therefore serve as an indisputable evidence of new physics [6,7]. We consider here two extensions of the SM which introduce sterile Majorana fermions. The mixing in the neutral lepton sector induced by these states also opens the possibility for flavour violation in $Z v_{i} v_{j}$ interactions (flavour-changing neutral currents), coupling both the left- and right- handed components of the neutral fermions to the $Z$ boson. Together with the charged-current LFV couplings, these interactions will induce an effective cLFV vertex $Z \ell_{1}^{\mp} \ell_{2}^{ \pm}$.

Inverse Seesaw The Inverse Seesaw (ISS) mechanism [8] is an example of low-scale seesaw realisation which in full generality calls upon the introduction of at least two generations of SM singlets. Here, we consider the addition of three generations of right-handed (RH) neutrinos $v_{R}$ and of extra $S U(2)$ singlets fermions $X$, to the SM particle content. Both $v_{R}$ and $X$ carry lepton number $L=+1$. The ISS Lagrangian reads $\mathscr{L}_{\mathrm{ISS}}=\mathscr{L}_{\mathrm{SM}}-Y_{i j}^{v} \bar{v}_{R i} \tilde{H}^{\dagger} L_{j}-M_{R i j} \bar{v}_{R i} X_{j}-\frac{1}{2} \mu_{X_{i j}} \bar{X}_{i}^{c} X_{j}+$ h.c. where $i, j=1,2,3$ are generation indices and $\tilde{H}=i \sigma_{2} H^{*}$. Lepton number $U(1)_{L}$ is broken only by the non-zero Majorana mass term $\mu_{X}$, while the Dirac-type RH neutrino mass term $M_{R}$ does conserve lepton number. In the $\left(v_{L}, v_{R}^{c}, X\right)^{T}$ basis, and after the electroweak symmetry breaking, the (symmetric) $9 \times 9$ neutrino mass matrix $\mathscr{M}$ is given by

$$
\mathscr{M}=\left(\begin{array}{ccc}
0 & m_{D}^{T} & 0 \\
m_{D} & 0 & M_{R} \\
0 & M_{R}^{T} & \mu_{X}
\end{array}\right),
$$

with $m_{D}=Y^{v} \mathrm{v}$ the Dirac mass term, v being the vacuum expectation value of the SM Higgs boson. Under the assumption that $\mu_{X} \ll m_{D} \ll M_{R}$, the diagonalization of $\mathscr{M}$ leads to an effective Majorana mass matrix for the active (light) neutrinos [9], $m_{v} \simeq m_{D}^{T} M_{R}^{T-1} \mu_{X} M_{R}^{-1} m_{D}$. The remaining six (mostly) sterile states form nearly degenerate pseudo-Dirac pairs. The possibility of having sizeable mixings between the active and sterile states, will have a non-negligible impact for several observables, thus rendering the ISS framework phenomenologically appealing. 
The effective " $3+\mathbf{1}$ model" A simpler approach to address the impact of sterile fermions on rare cLFV $Z$ decays consists in considering a minimal model where only one sterile Majorana state is added to the three light active neutrinos of the SM. This allows for a generic evaluation of the impact of the sterile fermions for these processes. In this simple toy model, no assumption is made on the underlying mechanism of neutrino mass generation. The addition of an extra neutral fermion to the particle content translates into extra degrees of freedom: the mass of the new sterile state $m_{4}$, three active-sterile mixing angles $\theta_{i 4}$, three new CP phases (two Dirac and one Majorana).

In our analysis, and for both hierarchies of the light neutrino spectrum, we scan over the following range for the sterile neutrino mass: $10^{-9} \mathrm{GeV} \lesssim m_{4} \lesssim 10^{6} \mathrm{GeV}$, while the active-sterile mixing angles are randomly varied in the interval $[0,2 \pi]^{1}$. All CP phases are also taken into account, and likewise randomly varied between 0 and $2 \pi$.

\section{Constraints on sterile neutrino extensions of the SM}

The introduction of sterile fermion states, which have a non-vanishing mixing to the active neutrinos, leads to a modification of the leptonic charged current Lagrangian:

$$
-\mathscr{L}_{\mathrm{cc}}=\frac{g}{\sqrt{2}} \mathbf{U}^{j i} \bar{\ell}_{j} \gamma^{\mu} P_{L} v_{i} W_{\mu}^{-}+\text {c.c. },
$$

where $\mathbf{U}$ is the leptonic mixing matrix, $i=1, \ldots, n_{v}$ denotes the physical neutrino states and $j=$ $1, \ldots, 3$ the flavour of the charged leptons. In the standard case of three neutrino generations, $\mathbf{U}$ corresponds to the unitary matrix $U_{\text {PMNS }}$. For $n_{v}>3$, the mixing between the left-handed leptons, which we denote by $\tilde{U}_{\text {PMNS }}$, corresponds to a $3 \times 3$ sub-block of $\mathbf{U}$, which can show some deviations from unitarity. One can parametrise [10] the $\tilde{U}_{\mathrm{PMNS}}$ mixing matrix as $U_{\mathrm{PMNS}} \rightarrow \tilde{U}_{\mathrm{PMNS}}=(\mathbb{1}-$ $\eta) U_{\text {PMNS }}$, where the matrix $\eta$ encodes the deviation of the $\tilde{U}_{\text {PMNS }}$ from unitarity $[11,12]$, due to the presence of extra neutral fermion states. One can also introduce the invariant quantity $\tilde{\eta}$, defined as $\tilde{\eta}=1-\left|\operatorname{Det}\left(\tilde{U}_{\mathrm{PMNS}}\right)\right|$, particularly useful to illustrate the effect of the new active-sterile mixings (corresponding to a deviation from unitarity of the $\tilde{U}_{\mathrm{PMNS}}$ ) on several observables.

The deviation from unitarity of $\mathbf{U}$ will induce a departure from the SM expected values of several observables. In turn, this is translated into a vast array of constraints which we will apply to our analysis (see details and references in [4]). Firstly, one has to ensure that the SM extension complies with $v$-oscillation data: we require compatibility with the corresponding best-fit intervals [13] (no constraints being imposed on the yet undetermined value of the $\mathrm{CP}$ violating Dirac phase $\delta$ ). We also apply unitarity bounds on the (non-unitary) matrix $\eta$; these arise from non-standard neutrino interactions with matter, and have been derived in $[14,15]$ by means of an effective theory approach (valid for sterile masses above the $\mathrm{GeV}$, but below the electroweak scale, $\Lambda_{\mathrm{EW}}$ ). We further take into account electroweak precision observables requiring, for instance, compatibility with LEP results on $\Gamma(Z \rightarrow v v)$. LHC data on invisible Higgs decays already allows to constrain regimes where the sterile states are below the Higgs mass. Negative laboratory searches for monochromatic lines in the spectrum of muons from $\pi^{ \pm} \rightarrow \mu^{ \pm} v$ decays also impose robust bounds on sterile neutrino masses in the $\mathrm{MeV}-\mathrm{GeV}$ range. The introduction of singlet neutrinos with Majorana masses allows

\footnotetext{
${ }^{1}$ We always ensure that the the perturbative unitary bound on the sterile masses and their couplings to the active states is respected.
} 
for new processes like lepton number violating interactions, among which neutrinoless double beta decay remains the most important one. In our analysis, we evaluate the contributions of the sterile states to the effective mass $m_{e e}$; we use the most recent constraint from EXO-200 (concerning future sensitivities we take $\left|m_{e e}\right| \lesssim 0.01 \mathrm{eV}$ ). Further constraints arise from leptonic and semileptonic decays of pseudoscalar mesons $K, D, D_{s}, B$. Recent studies suggest that in the framework of the SM extended by sterile neutrinos the most severe bounds arise from the violation of lepton universality in leptonic kaon decays. Other than the rare decays occurring in the presence of nuclei, the new states can contribute to several charged lepton flavour violating processes such as $\ell \rightarrow \ell^{\prime} \gamma$, $\ell \rightarrow \ell_{1} \ell_{1} \ell_{2}$. In our analysis we compute the contribution of the sterile states to all these observables imposing compatibility with the current experimental bounds. Finally, a number of cosmological observations [2] put severe constraints on sterile neutrinos with a mass below the TeV.

\section{Results}

\section{cLFV $Z$ decays in the ISS}

We show the results for this well motivated framework of neutrino mass generation in Fig. 1. On the left, we show the $\operatorname{BR}(Z \rightarrow \mu \tau)$ as a function of the average of the absolute masses of the mostly sterile states, $\left\langle m_{4-9}\right\rangle=\sum_{i=4 \ldots 9} \frac{1}{6}\left|m_{i}\right|$. In the plot on the left, we identify as grey points the solutions which fail to comply with (at least) one of the constraints listed in Section 3. We depict in red the points that survive all other bounds but are typically disfavoured from standard cosmology arguments. Finally, blue points are in agreement with all imposed constraints. These results indicate that this ISS realisation can account for sizeable values of cLFV Z-decay branching ratios, at least for the second and third generations of leptons, but mostly for cosmological disfavoured solutions. This in general requires sterile states with a mass $\gtrsim \Lambda_{\mathrm{EW}}$, and can occur even for very

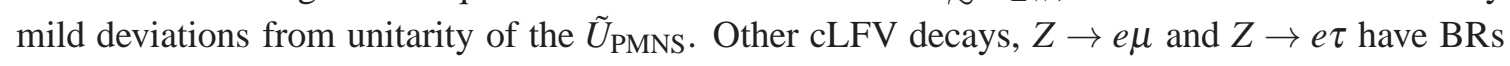
$\lesssim \mathscr{O}\left(10^{-11}\right)$, but still within experimental sensitivity. The prospects for the observation of cLFV $Z$ decays in this framework are summarised in the right plot of Fig. 1 by considering the values of $\mathrm{BR}\left(Z \rightarrow \ell_{1}^{\mp} \ell_{2}^{ \pm}\right)$in the $\left(\tilde{\eta},\left\langle m_{4-9}\right\rangle\right)$ parameter space of this specific realisation and for a NH light neutrino spectrum.

\section{cLFV $Z$ decays in the " $3+1$ model"}

This minimal extension of the SM by one sterile neutrino can account for values of $\operatorname{BR}\left(Z \rightarrow \ell_{1}^{\mp} \ell_{2}^{ \pm}\right)$ within the sensitivity of a high luminosity $Z$-factory, such as the FCC-ee. Nevertheless, the largest cLFV $Z$ decay branching fractions (as large as $\mathscr{O}\left(10^{-6}\right)$ ) cannot be reconciled with current bounds on low-energy cLFV processes. Indeed, sterile neutrinos also contribute via $Z$ penguin diagrams to cLFV 3-body decays and $\mu-e$ conversion in nuclei, which severely constrain the flavour violating $Z \ell_{1}^{\mp} \ell_{2}^{ \pm}$vertex (see also [7]). Moreover, the recent MEG result on $\mu \rightarrow e \gamma$ also excludes important regions of the parameter space. These constraints are especially manifest in the case of $Z \rightarrow e \mu$ decays, since the severe limits from $\operatorname{BR}(\mu \rightarrow 3 e)$ and $\mathrm{CR}(\mu-e, \mathrm{Au})$ typically preclude $\mathrm{BR}(Z \rightarrow$ $e \mu) \gtrsim 10^{-13}$. In Fig. 2 we illustrate the complementary rôle of a high-luminosity $Z$-factory with respect to low-energy (high-intensity) cLFV dedicated experiments (same color code as in Fig. 1). We display the sterile neutrino contributions to $\operatorname{BR}\left(Z \rightarrow \ell_{1}^{\mp} \ell_{2}^{ \pm}\right)$versus two different low-energy cLFV observables: $\mathrm{CR}(\mu-e, \mathrm{Al})$ and $\mathrm{BR}(\tau \rightarrow \mu \gamma)$. We further highlight in dark yellow solutions 

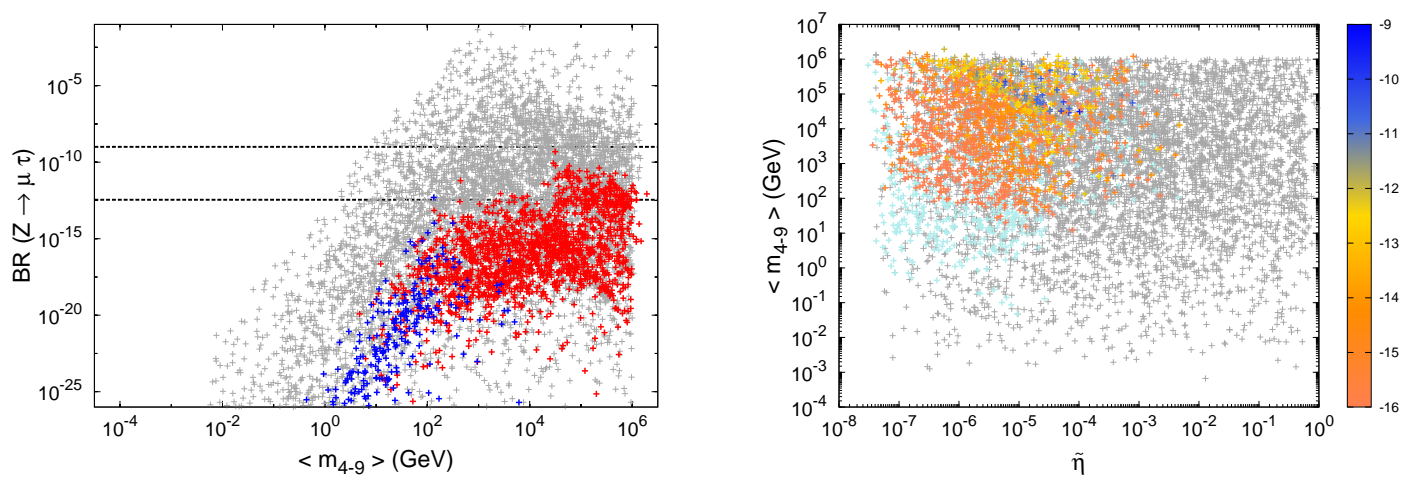

Figure 1: ISS realisation: $\operatorname{BR}(Z \rightarrow \mu \tau)$ as a function of the average value of the mostly sterile state masses (right), $\left\langle m_{4-9}\right\rangle$ (see text for the description of the color code), for a NH light neutrino spectrum (left); maximal values (in log scale) of $\mathrm{BR}\left(Z \rightarrow \ell_{1}^{\mp} \ell_{2}^{ \pm}\right)$on the $\left(\tilde{\eta},\left\langle m_{4-9}\right\rangle\right)$ parameter space (right) for a NH light neutrino spectrum, from larger (dark blue) to smaller (orange) values. Cyan denotes values of the branching fractions below $10^{-18}$.

which allow for a third complementary observable within future sensitivity, which is the effective neutrino mass in $0 v 2 \beta$ decays.
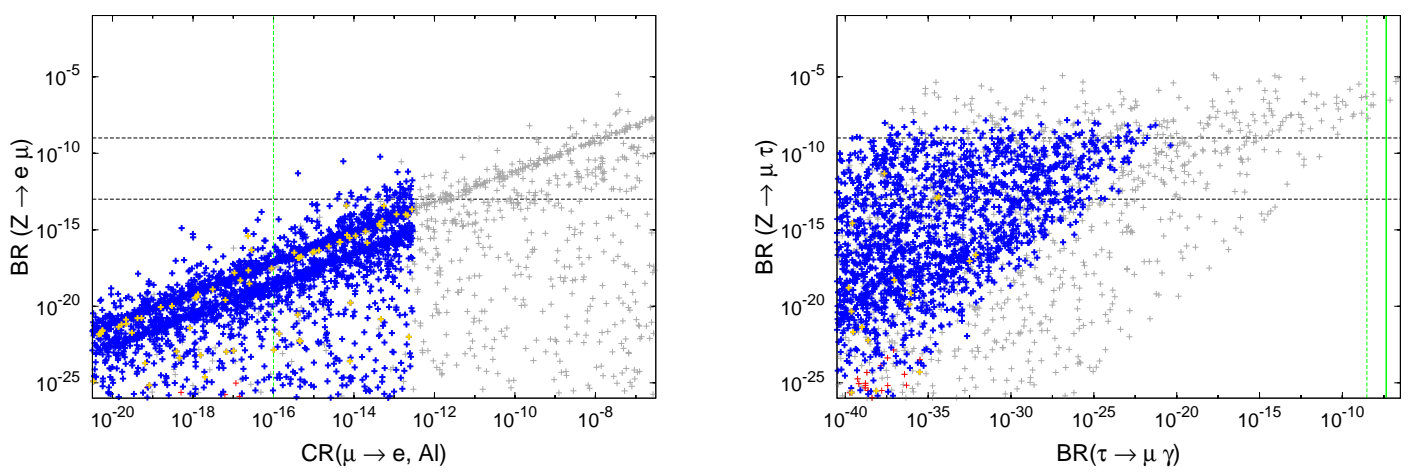

Figure 2: The " $3+1$ model": on the left, $\mathrm{BR}(Z \rightarrow e \mu)$ versus $\mathrm{CR}(\mu-e, \mathrm{Al})$, and $\mathrm{BR}(Z \rightarrow \mu \tau)$ vs $\mathrm{BR}(\tau \rightarrow \mu \gamma)$ on the right, for a NH light neutrino spectrum (IH leads to similar results). See text for the description of the color code. When present, the additional green vertical lines denote the current bounds (solid) and future sensitivity (dashed), and dark-yellow points denote an associated $\left|m_{e e}\right|$ within experimental reach.

As can be inferred from Fig. 2, low-energy cLFV dedicated facilities offer much better prospects to probe LFV in the $\mu-e$ sector of the " $3+1$ model" than a high-luminosity $Z$-factory. In particular, Mu3e (PSI) [16] and COMET (J-PARC) [17] will be sensitive to regions in parameter space associated with $\operatorname{BR}(Z \rightarrow e \mu) \sim 10^{-17 \div-13}$, beyond the reach of FCC-ee. Interestingly, the situation is reversed for the case of the $\mu-\tau$ sector. Moreover, a non negligible subset of the parameter space is testable at a third type of facilities, through $0 v 2 \beta$ decay searches (especially in the case of an IH light neutrino spectrum, although we have not displayed it here).

\section{Conclusions}

We have considered two extensions of the SM which add to its particle content one or more sterile 
neutrinos. We have explored indirect searches for these sterile states at a future circular collider like FCC-ee, running close to the $Z$ mass threshold. We have considered the contribution of the sterile states to rare cLFV $Z$ decays in these two classes of models and discussed them taking into account a number of experimental and theoretical constraints. Among these, low-energy LFV observables like cLFV 3-body decays and $\mu-e$ conversion in nuclei impose strong constraints on the sterile neutrino induced $\operatorname{BR}\left(Z \rightarrow \ell_{1}^{\mp} \ell_{2}^{ \pm}\right)$. Our analysis emphasises the underlying synergy between a high-luminosity $Z$ factory and dedicated low-energy facilities: regions of the parameter space of both models can be probed via LFV $Z$ decays at FCC-ee, at low-energy cLFV dedicated facilities and also via searches for $0 v 2 \beta$. Notably, FCC-ee could better probe LFV in the $\mu-\tau$ sector, in complementarity to the reach of low-energy experiments like COMET.

\section{Acknowledgments}

We acknowledge support from the EU FP7 ITN INVISIBLES (Marie Curie Actions, PITN-GA-2011-289442).

\section{References}

[1] K. N. Abazajian et al., arXiv:1204.5379 [hep-ph].

[2] A. Kusenko, Phys. Rept. 481 (2009) 1 [arXiv:0906.2968 [hep-ph]].

[3] M. Bicer et al., JHEP 1401 (2014) 164 [arXiv:1308.6176 [hep-ex]].

[4] A. Abada et al., JHEP 1504 (2015) 051 [arXiv:1412.6322 [hep-ph]].

[5] S. L. Glashow et al., Phys. Rev. D 2 (1970) 1285.

[6] G. Mann and T. Riemann, Annalen Phys. 40 (1984) 334; J. I. Illana et al., in 2nd ECFA/DESY Study 1998-2001, 490-524 [hep-ph/0001273]; J. I. Illana and T. Riemann, Phys. Rev. D 63 (2001) 053004 [hep-ph/0010193]; A. Ilakovac and A. Pilaftsis, Nucl. Phys. B 437 (1995) 491 [hep-ph/9403398]; S. Davidson, S. Lacroix and P. Verdier, JHEP 1209 (2012) 092 [arXiv:1207.4894 [hep-ph]].

[7] M. A. Perez et al., Int. J. Mod. Phys. A 19 (2004) 159 [hep-ph/0305227]; A. Flores-Tlalpa et al., Phys. Rev. D 65 (2002) 073010 [hep-ph/0112065]; D. Delepine and F. Vissani, Phys. Lett. B 522 (2001) 95 [hep-ph/0106287].

[8] R. N. Mohapatra and J. W. F. Valle, Phys. Rev. D 34 (1986) 1642.

[9] M. C. Gonzalez-Garcia and J. W. F. Valle, Phys. Lett. B 216 (1989) 360.

[10] E. Fernandez-Martinez et al., Phys. Lett. B 649 (2007) 427 [hep-ph/0703098].

[11] J. Schechter and J. W. F. Valle, Phys. Rev. D 22 (1980) 2227.

[12] M. Gronau, C. N. Leung and J. L. Rosner, Phys. Rev. D 29 (1984) 2539.

[13] D. V. Forero et al., Phys. Rev. D 90 (2014) 093006 [arXiv:1405.7540 [hep-ph]].

[14] S. Antusch et al., Nucl. Phys. B 810 (2009) 369 [arXiv:0807.1003 [hep-ph]].

[15] S. Antusch and O. Fischer, JHEP 1410 (2014) 94 [arXiv:1407.6607 [hep-ph]].

[16] A. Blondel et al., arXiv:1301.6113 [physics.ins-det].

[17] Y. Kuno [COMET Collaboration], PTEP 2013 (2013) 022C01. 